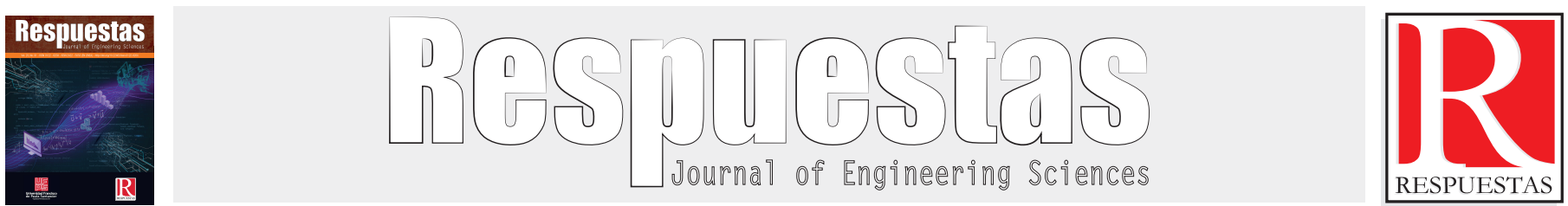

Artículo Original

https://doi.org/10.22463/0122820X.1505

\title{
Incidencia de la cultura y la condición socioeconómica en el proceso de enseñanza - aprendizaje desarrollado en las ciencias empresariales
}

\author{
Influence of culture and socio-economic status on the teaching - learning process developed in the business sciences \\ Gerson Rueda Vera. \\ ${ }^{a^{*}}$ Magister en Práctica Pedagógica, gersonruedavera@ufps.edu.co,orcid.org/0000-0001-9032-7100, Universidad Francisco de Paula Santander, Cúcuta, Colombia
}

How to cite: G. Rueda-Vera, "Incidencia de la cultura y la condición socioeconómica en el proceso de enseñanza - aprendizaje desarrollados en la ciencias empresariales" Respuestas, vol. 23, no. S1, pp. 70-75, 2018. https://doi.org/10.22463/0122820X.1505

Recibido: Febrero 10, 2018; Aprobado: Junio 30, 2018.

\begin{tabular}{ll}
\hline & RESUMEN \\
\hline Palabras Clave: & El objetivo de la investigación se centró en conocer si las variables: conocimiento de la interculturalidad, lugar de residencia, \\
Educación & percepción de igualdad, y la realidad socioeconómica, son relevantes para el proceso enseñanza-aprendizaje en los programas \\
Inclusión & de ciencias empresariales de la Universidad Francisco de Paula Santander (Cúcuta-Colombia) se abordó desde lo rurbano y la \\
Intercultura & educación intercultural, con una metodología cuantitativa, de estudio descriptivo correlacional, con la encuesta como \\
Rurbano & instrumento de recolección de datos, aplicada a 105 docentes y 420 estudiante, Resultados. Existe un conocimiento del tema, \\
& hay poca preocupación por conocer el contexto del estudiante, se conserva un espacio de dialogo sin discriminación dentro de \\
& las aulas. Conclusión: Es necesario mejorar las orientaciones que están direccionadas al perfeccionamiento de las habilidades y \\
& destrezas que pueda adquirir el sujeto para el desarrollo de una profesión, indistintamente de su procedencia, nivel social y \\
& cantidad de recursos que posea.
\end{tabular}

\section{ABSTRACT}

Keywords:

The aim of the research centred on knowing if the variables: knowledge of the interculturalidad, place of residence, the perception of equality, and socioeconomic reality are relevant for the process education - learning in the programs of managerial sciences of the University Francisco de Paula Santander (Cúcuta-Colombia) approached from rurbano and the intercultural education, with a quantitative methodology, of descriptive study correlacional, with the survey as instrument of compilation of information, applied to 105 teachers and 420 student, Resultados. A knowledge of the topic exists, there is few worry for knowing the context of the student, a space of dialog remains without discrimination inside the classrooms. Conclusion: It is necessary to improve the guidelines that are aimed at perfecting the skills and abilities that the subject can acquire for the development of a profession, regardless of their origin, social level and amount of resources they have.

\section{Introducción}

Al dar una mirada a la historia se puede resaltar que en épocas anteriores acceder a la educación era para las clases más privilegiadas, esta situación de manera indirecta hizo que se marginara a las clases menos favorecidas, creando unos hábitos y costumbres distintos aun cuando residían en las mismas zonas, esto generó una brecha entre el diario vivir de un empleado o campesino frente a los miembros de las familias adineradas, esta situación se dio en todas las naciones creando así unas castas o estratos sociales, [1] se conoce que alrededor de 40 de los 115 millones de niños y niñas que están fuera de la escuela en el mundo; por otra parte, al observar la realidad de américa latina puede referenciarse que existe diversidad en la cultura, debido a las mezclas creadas por los diferentes grupos étnicos y los migrantes que colonizaron esta área geográfica.

La educación debe estar pensada desde el cumplimiento a las necesidades de los estudiantes sin distingo de las característi- cas, limitaciones o capacidades, [2] la diversidad es un proceso amplio y dinámico de construcción y reconstrucción del conocimiento, a partir del intercambio y el dialogo entre personas con diferentes valores, ideas, percepciones, intereses, capacidades y estilos de aprendizaje, en este sentido [3] se precisa el concepto de diversidad como "un rasgo definitorio de las sociedades contemporáneas, fruto principalmente de los movimientos migratorios de la sociedad global".

Si se contempla la diversidad como la complejidad de las situaciones sociales y educativas, es necesario comprender que la forma como pensamos o los imaginarios con relación al entrono, hace exista una forma particular al hablar o actuar, esta realidad se puede percibir en los individuos pertenecientes a los sectores urbanos situados en las periferias de la ciudad de Cúcuta, quienes convergen en las diferentes instituciones educativas del municipio, convirtiendo la Universidad Francis-

*Autor de correspondencia.

E-mail: gersonruedavera@ufps.edu.co (Gerson Rueda Vera),

cc) (i) (s) $\odot$ Peer review is the responsibility of the Universidad Francisco de Paula Santander cc) (1) () $($ Th 
co de Paula Santander un espacio multicultural, multiétnico y que alberga estudiantes de diferentes condiciones sociales, que a su vez permea los programas de la facultad de ciencias empresariales.

\section{La interculturalidad y lo rurbano}

La complejidad social y los diferentes escenarios de la educación han posibilitado el reflexionar sobre los cambios que han surgido, [4] en la actualidad la sociedad está compuesta por diversas corrientes del pensamiento y un cumulo de saberes, los cuales han sufrido una separación, donde predomina la ceguera por consecuencia de los saberes parcelados y dispersos, propios de una visión unidimensional de todas las cosas, generando espejismos de la sociedad del conocimiento y del pleno empleo de la racionalidad. [5]

Los orígenes de los individuos son fundamentales, donde se puede afirmar que la cultura enraizada en la sociedad occidental ha sufrido transformación pero siempre seguida de las fórmulas represivas de la racionalidad positivista, donde el crecimiento dominante de lo racional se ha extendió a las instituciones educativas.

En relación al proceso de enseñanza, [6] se manifiesta lo siguiente: La formación debe ser permanente y si en esta experiencia se acepta que quien imparte el conocimiento es el sujeto en relación frente al cual los estudiantes se consideran el objeto, entonces el sujeto que forma es el maestro y los estudiantes son el objeto formado por él, donde, se aprecia el individuo como un recipiente que recibe los conocimientos acumulados por el sujeto y que son transferidos en el proceso de enseñanza.

Esta situación sigue dándose en los procesos educativos impidiendo la participación; por tal razón, [7] es necesario abrir espacios donde se argumenta de forma reflexiva, donde se tenga en cuenta los cambios culturales para contextualizar este proceso y que se apoye en elementos como la diversidad y el diálogo, convirtiéndose en un punto de partida para fortalecer la educación intercultural que surge de las necesidades de la sociedad y la voluntad política para abordad los conflictos internos, que son originados por las diferencias culturales y socioeconómicas.

Se puede decir que la aparición de interculturalidad [8] surge a partir de dos experiencias sociopolíticas de aproximación a la diversidad cultural, la primera es la multiculturalista y la segunda es la asimilacionista, estas anteceden al ideal de la intercultura que es hacia donde están orientados los esfuerzos de la sociedad; además en cuanto a esta nueva corriente educativa, [9] es de resaltar la importancia del intercambio cultural y el respeto, en donde todos los individuos tiene derecho a la participación sin importar su origen, procedencia o condición social, aunque en nuestra sociedad es difícil legitimar este término debido al poder y hegemonía ejercida por un grupo respecto a otro.

De conformidad con el tema de la interculturalidad [10] desde el plano de las competencias y las aproximaciones críticas y analíticas, se establece lo siguiente: A partir de las experiencias previas, así como, el contexto del que provenga el sujeto, de lo que observa en sus actividades cotidianas y del proceso de formación construye el conocimiento y el significado o conceptualización de la realidad.[11] lo anterior se refiere a la diversidad cultural, religiosa, lingüística, social, de sexo, ligada a factores interpersonales, de acceso al conocimiento, y de necesidades educativa.

La educación inclusiva establece un espacio donde se valora y respeta a todos, generando una permanente reflexión de lo que se enseña y como se enseña, [12] una de las condiciones necesarias para el desarrollo de escuelas inclusivas es que los profesores compartan una serie de valores, creencias y actitudes acordes con los principios de la inclusión y que concreten dichos pensamientos en una enseñanza orientada al logro de la participación plena de los alumnos en un entorno educativo normalizado.

Las iniciativas sobre inclusión en educación deben ser analizadas en relación a las políticas interna de acceso de las instituciones de educación, quienes determinan la aceptación de los estudiantes, siendo estas las encargadas de permitir el ingreso de estudiantes indistintamente la cultura, creencias o su localización geográfica.

En los municipios los espacios de "borde" han sido escenarios de trasformaciones sociales, la constante migración campo-ciudad y habitantes de bajos recursos lo han configurado entre un contexto citadino y uno rural. [13], las personas que habitan el espacio de "borde rurbano" son: "lo imprevisto y lo no deseado de la ciudad, lo que se quiere borrar, alejar, desalojar, transferir, transportar, volver invisible. En cierta forma se da un proceso de translocación de los conocimientos y prácticas culturales donde se anudan los nuevos estereotipos que rodean a los espacios rurbanos generando nuevas identidades.

En cuanto a la población que se establece en los sectores determinados como rurbanos se caracterizan por ser [14] un conjunto heterogéneo de personas de bajos recursos, que en algunas ocasiones provienen del interior del país o de zonas rurales cercanas, y en el caso de los jóvenes de este grupo que acceden a la educación poseen una condición particular puesto que alguna veces deben trabajar, son madres cabeza de familia, están casados, pertenecen a grupos indígenas, o tienen alguna discapacidad. Se debe aceptar que hay que afrontar este fenómeno por parte de las instituciones educativas, pero, [15] desafortunadamente, el predominio de ciertos imaginarios y prejuicios respecto a estos grupos poblacionales generan temor en algunas instituciones, asumiendo que con el ingreso de estos 
sujetos podrían convertirse potencialmente en un problema más que enfrentar, y no se piensa como un acto de responsabilidad social y una oportunidad de aprendizaje institucional para estos integrantes de la comunidad.

\section{Materiales y métodos}

En cuanto al enfoque o paradigma [16] el cuantitativo utiliza la recolección y el análisis de datos confiando en la medición numérica, el conteo y frecuentemente en el uso de la estadística para establecer con exactitud patrones de comportamiento de una población. Por lo anterior expuesto el paradigma investigativo es cuantitativo. El diseño investigativo [17] se define como el plan global de investigación que intenta de una manera clara dar respuestas a las preguntas planteadas en la misma". El desarrollo del proyecto se hizo de forma descriptiva. La población la componen individuos que poseen una característica común, [18] "está determinada por el conjunto de elementos que poseen unas características definitorias". En el proyecto la población está dada por 144 Docente y 5320 Estudiantes de la facultad de ciencias empresariales. En cuanto a la Muestra, [19] permite al investigador seleccionar un subgrupo de unidades de la población a las que se les requerirá información, dichos individuos deben representar universo objeto de estudio. La selección de la muestra fue de manera probabilística y como resultado el grupo lo conforman 105 docentes y 420 estudiantes. Para la recolección de la información se diseñó un instrumento [20] el cual se define como una serie de preguntas que debe ser respondido por un individuo, sin la intervención de quien lo aplica. Para la investigación se utilizó la encuesta, se validó estadísticamente con la prueba alfa de Cronbach y adicionalmente por juicio de pares expertos.

\section{Resultados y análisis}

La información que se presenta a continuación es la representación analítica a partir de la utilización de métodos estadísticos en el procesamiento de la información que se obtuvo de docente y estudiantes que pertenecen a los programas de la facultad de ciencias empresariales de la UFPS.

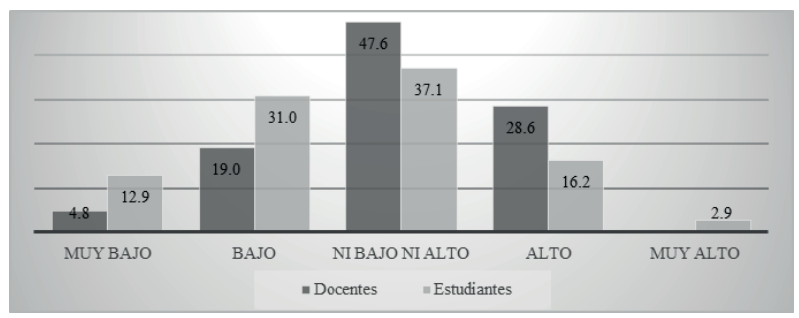

Figura 1. ¿Cómo es el conocimiento que tiene en cuanto a la interculturalidad?
Es de resaltar que las respuestas obtenidas reflejan ciertas variaciones, donde las concepciones de los docentes deja entrever que definitivamente les falta a la totalidad de ellos profundizar en el tema, sin desconocer que casi la totalidad de las respuestas están marcados en un conocimiento medio, además los maestro poseen un porcentaje muy pequeño que manifiesta tener un conocimiento muy bajo, a diferencia de las respuestas de estudiantes, donde hay un pequeño número que responde tener muy alto conocimiento de la interculturalidad, pero de forma antagónica se observa que un porcentaje representativo dicen tener muy bajo conocimiento del tema.

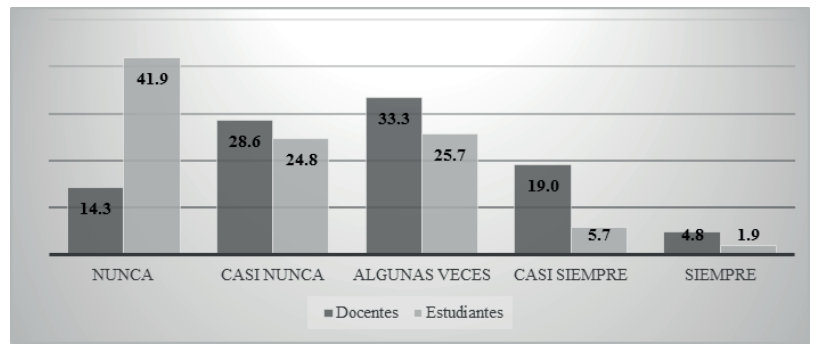

Figura 2. ¿Con qué frecuencia tiene en cuenta la ubicación de residencia de los estudiantes para asignar los trabajos extracurriculares?

Recopilando las respuestas de los participantes en relación a la pregunta se revela una congruencia, puesto que son muy pocas las diferencias entre las respuestas de docentes y estudiantes, la mayoría de ellos afirman que casi nunca o de forma eventual es tenido en cuenta los estratos sociales para la asignación de actividades complementarias, sin desconocer que existe un grupo pequeño que opina que siempre que se establecen actividades extracurriculares un elemento relevante es conocer los lugares de residencias para la asignación de actividades adicionales a la asignatura.

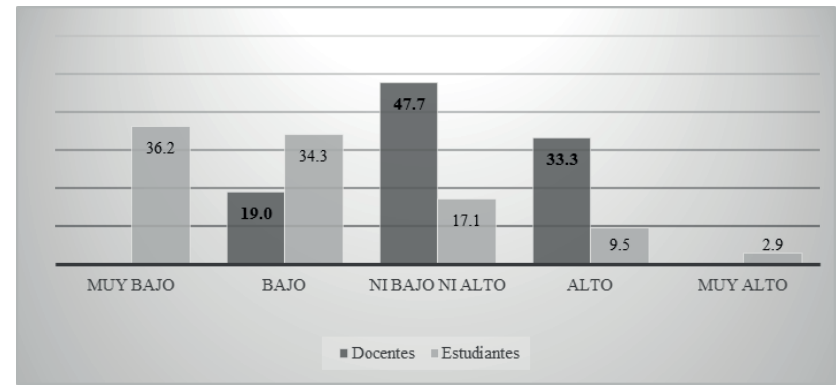

Figura 3. ¿Qué tanto conocimiento tiene de las condiciones socio-económicas de los estudiantes que integran las asignaturas que orienta?

Al realizar la interpretación de los datos graficados se observa en la respuesta de los docentes que poseen un mediano conocimiento de la realidad que viven los estudiantes, por otra parte, la percepción que tienen los estudiantes en cuanto si los docentes conocen sus condiciones socio-económica, más de la mitad de ellos consideran que los maestros ignoran las dificul- 
tades que poseen, la anterior afirmación se respalda con los porcentajes estadísticos mayoritarios representados en las opciones de respuestas bajo y muy bajo.

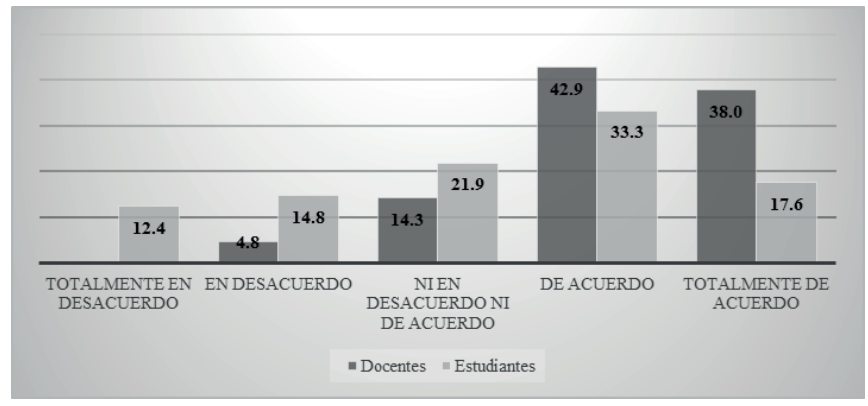

Figura 4. ¿En el aula existe un espacio de dialogo sin importar la condición social o económica del estudiante?

Con referencia a la pregunta existe concordancia en el comparativo puesto que son muy pocas las diferencias entre las respuestas de docentes y estudiantes, se debe resalta que más del $80 \%$ de los docentes está de acuerdo y totalmente que existe un espacio de dialogo en el aula de clase; por otra partes más de la mitad de los estudiantes respondieron que existe ese espacio donde poder exponer sus ideas, aunque hay un $12,4 \%$ de los estudiantes que sostienen estar totalmente en desacuerdo con esta afirmación y para ello no es posible sostener un dialogo en el aula de clase.

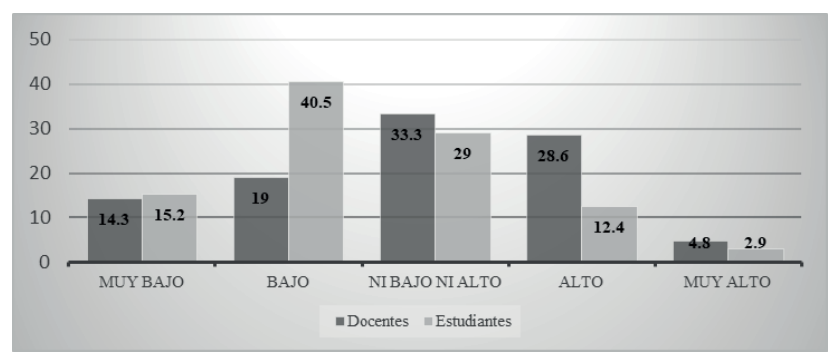

Figura 5. ¿Qué tanto conocimiento tienen los docentes referente a los hábitos culturales de los estudiantes a su cargo?

Se puede deducir que a los docentes les falta mayor conocimiento acerca de los hábitos que tienen las diferentes culturas representadas en el aula de clase, además al interpretar la respuesta de los estudiantes la mayoría de ellos perciben el bajo conocimiento que tienen sus maestro de las características regionales o rurbanas que poseen, indicando que la diversidad cultural está presente y es una realidad generando un nuevo escenario donde el profesor debe aumentar el conocimiento al respecto del tema para que el proceso de enseñanza-aprendizaje tenga un mayor impacto en la formación de futuros profesionales.

Es necesario conocer si las variables tienen incidencia entre ellas y en el desarrollo de la actividad docente al realizar el proceso de enseñanza aprendizaje, para ello se correlacionaron con el propósito de saber si hay significancia estadística entre ellas.

Tabla I. Medidas simétricas de correlación entre los ítems 1 y 2

\begin{tabular}{|c|c|c|c|c|c|}
\hline \multicolumn{2}{|c|}{} & Valor & Error típ. asint $^{*}$ & T aproximada $^{\text {b }}$ & Sig. Aproximada \\
\hline \multirow{2}{*}{$\begin{array}{c}\text { Ordinal por } \\
\text { ordinal }\end{array}$} & Tau-b de Kendall &, 239 &, 039 & 6,049 &, 000 \\
\cline { 2 - 6 } & Gamma &, 333 &, 054 & 6,049 &, 000 \\
\cline { 2 - 6 } & $\begin{array}{c}\text { Correlación de } \\
\text { Spearman }\end{array}$ &, 279 &, 046 & 5,946 &, $000^{\text {c }}$ \\
\hline $\begin{array}{c}\text { Intervalo por } \\
\text { intervalo }\end{array}$ & R de Pearson &, 249 &, 048 & 5,258 &, $000^{\text {c }}$ \\
\hline \multicolumn{2}{|c|}{ N de casos válidos } & 420 & & & \\
\hline
\end{tabular}

Se refleja una correlación con un valor $\mathrm{p}<0.05$, lo que se traduce en correlación positiva que significa la asociación entre el conocimiento que se tiene en cuanto a la interculturalidad versus la frecuencia en que los docentes tienen en cuenta la ubicación del lugar de residencia de los estudiantes para los trabajos extracurriculares asignado, esto indica que es necesario apropiarse mas del tema de diversidad cultural para comprender las condiciones sociales relacionadas al rendimiento académico en función de la maximización de los recurso que poseen los estudiantes.

Tabla II. Medidas simétricas de correlación entre los ítems 1 y 3

\begin{tabular}{|c|c|c|c|c|c|}
\hline \multicolumn{2}{|c|}{} & Valor & Error típ. asint $^{\mathrm{a}}$ & T aproximada & Sig. Aproximada \\
\hline \multirow{3}{*}{$\begin{array}{c}\text { Ordinal por } \\
\text { ordinal }\end{array}$} & Tau-b de Kendall &, 201 &, 039 & $\mathbf{5 , 1 5 0}$ &, 000 \\
\cline { 2 - 6 } & Gamma &, 276 &, 052 & 5,150 &, 000 \\
\cline { 2 - 6 } & $\begin{array}{c}\text { Correlación de } \\
\text { Spearman }\end{array}$ &, 239 &, 046 & $\mathbf{5 , 0 3 2}$ & $\mathbf{0 0 0}^{\mathrm{c}}$ \\
\hline $\begin{array}{c}\text { Intervalo por } \\
\text { intervalo }\end{array}$ & R de Pearson &, 233 &, 048 & 4,900 & $\mathbf{0 0 0}^{\mathrm{c}}$ \\
\hline \multicolumn{2}{|c|}{ N de casos válidos } & 420 & & & \\
\hline
\end{tabular}

Se refleja una correlación con un valor $\mathrm{p}<0.05$, lo que se traduce en una correlación positiva la cual simboliza que, existe una asociación entre el saber que se tiene en cuanto a la interculturalidad confrontado con el conocimiento que tienen los docentes de las condiciones socio-económicas de los estudiantes que integran las asignaturas que orienta, esto indica que los docentes poseen poco conocimiento referente al tema, además no perciben como importante indagar las condiciones socio-económicas del grupo de estudiantes a su cargo para así promover un mayor rendimiento académico.

Tabla III. Medidas simétricas de correlación entre los ítems 3 y 4

\begin{tabular}{|c|c|c|c|c|c|}
\hline \multicolumn{2}{|c|}{} & Valor & Error típ. asint $^{\mathrm{a}}$ & T aproximada $^{\mathrm{b}}$ & Sig. Aproximada \\
\hline \multirow{2}{*}{$\begin{array}{c}\text { Ordinal por } \\
\text { ordinal }\end{array}$} & Tau-b de Kendall &, 115 &, 043 & 1,681 &, 007 \\
\cline { 2 - 6 } & Gamma &, 152 &, 056 & 1,681 &, 007 \\
\cline { 2 - 6 } & $\begin{array}{c}\text { Correlación de } \\
\text { Spearman }\end{array}$ &, 140 &, 051 & 1,899 &, $0004^{\text {c }}$ \\
\hline $\begin{array}{c}\text { Intervalo por } \\
\text { intervalo }\end{array}$ & R de Pearson &, 171 &, 051 & 2,538 & $\mathbf{, 0 0 0}^{\text {c }}$ \\
\hline \multicolumn{2}{|c|}{ N de casos válidos } & 420 & & & \\
\hline
\end{tabular}


Se refleja una correlación con un valor $\mathrm{p}>0.05$, expresando así que es una correlación no significativa, y simboliza entonces no existe asociación o relación entre las variables: Que tanto conocimiento tiene de las condiciones socio-económicas de los estudiantes que integran las asignaturas que orienta, versus a la existencia de un espacio de dialogo en el aula sin importar la ubicación de domicilio o la procedencia del estudiante, es decir los docentes fomentan la participación con el propósito de fundamentar los temas académicos propios de la profesión, indistintamente del conocimiento que tenga con respecto a los factores económicos, sociales o familiares de los estudiantes que orienta.

Tabla IV. Medidas simétricas de correlación entre los ítems 1 y 5

\begin{tabular}{|c|c|c|c|c|c|}
\hline & & Valor & Error típ. asint ${ }^{a}$ & $T$ aproximada ${ }^{b}$ & Sig. Aproximada \\
\hline \multirow{3}{*}{$\begin{array}{c}\text { Ordinal por } \\
\text { ordinal }\end{array}$} & Tau-b de Kendall & ,413 & ,040 & 9,942 & ,000 \\
\hline & Gamma &, 549 & ,050 & 9,942 & ,000 \\
\hline & $\begin{array}{c}\text { Correlación de } \\
\text { Spearman }\end{array}$ & 4,465 & ,045 & 10,747 &, $000^{c}$ \\
\hline $\begin{array}{c}\text { Intervalo por } \\
\text { intervalo }\end{array}$ & R de Pearson &, 482 & ,045 & 11,254 &, $000^{\mathrm{c}}$ \\
\hline \multicolumn{2}{|c|}{$\mathrm{N}$ de casos válidos } & 420 & & & \\
\hline
\end{tabular}

Se refleja una correlación con un valor $\mathrm{p}<0.05$, lo que se traduce en correlación positiva, lo cual simboliza la asociación entre las variables correspondientes al saber que el docentes tiene de las condiciones socio-económicas de los estudiantes comparado con la pregunta ¿Qué tanto conocimiento tienen los docentes de los hábitos culturales de los estudiantes a su cargo?, se refleja entonces que es necesario aumentar las conceptualizaciones de intercultura o de la diversidad cultural y lo rurbano para comprender como es el comportamiento y el nivel de rendimiento académico de los estudiantes que hacen parte del grupo que orienta a partir de los hábitos que son inherente a las regiones y condiciones sociales a las que ellos pertenecen.

El concepto de lo rurbano cambia de forma radical el procesos enseñanza aprendizaje, puesto que está ligado a las zonas en donde se domicilian los jóvenes que hacen parte de la institución educativa, lo que hace que la realidad del quehacer docente cambie abruptamente puesto que existen hábitos particulares de otras regiones y que son comunes en los asentamientos o colonias que se establecen en los bordes de la ciudad, y los sujetos de la comunidad los toman como propios; adicionalmente, dentro del análisis de la información se pudo observar que en respuesta de los encuestados que hay un nivel medio de conocimiento en relación a la diversidad de condiciones sociales de los estudiantes que conforman el grupo, es por ello que un porcentaje significativo de docentes, algunas veces o casi nunca tienen en cuentas la condición social de los alumnos para el desarrollo de actividades que implique recursos económicos.

En cuanto a la cultura se realizó un sondeo para observar el respeto para con los jóvenes que hacen parte de la facultad de
Ciencias Empresariales de la UFPS, para ello se preguntó ¿En el aula existe un espacio de dialogo sin importar la ubicación de domicilio o la procedencia del estudiante? Y la mayor cantidad de respuestas son: De acuerdo y totalmente de acuerdo.

Otro ítem aplicado a la muestra estadística es ¿Qué tanto conocimiento tiene los docentes de los hábitos culturales de los estudiantes a su cargo? Y tanto docentes como estudiantes concordaron en las respuestas Bajo y ni bajo ni alto.

Una de las ideas centrales de la investigación es la educación intercultural y para ello se consultó a docentes y estudiantes a través del interrogante ¿Considera que las políticas educativas tienen en cuenta la diversidad cultural y la condición socio-económica de los estudiantes? Los porcentajes más altos son las respuestas: En desacuerdo, así como, $\mathrm{Ni}$ en desacuerdo ni de acuerdo. Es necesario conocer si existe inclusión para todos los individuos objeto de estudio, para ello se realizó el siguiente cuestionamiento ¿En qué ocasiones ha tenido que adquirir materiales costosos o fuera del alcance socio-económico para cumplir con el contenido de una asignatura? Y se obtuvo que las respuestas más representativas son: Casi nunca $\mathrm{y}$ algunas veces.

\section{Conclusiones}

La educación debe tener características que permitan dentro de su estructura ser incluyente, donde la condición de rurbanidad sea reconocida ya que esta torga el reconocimiento de los habitantes de borde de área del municipio, la realidad que se observa al interior de la facultad de ciencias empresariales de la UFPS, es que la mayoría de los docentes tiene conocimiento sobre la interculturalidad y como se presenta en el aula de clase, además, reconocen la realidad de las condiciones socio-económicas que poseen los estudiantes que integran las asignaturas que orienta, de igual manera, el proceso de enseñanza - aprendizaje tiene una connotación particular que es resultado de las diversas condiciones sociales, siendo cada grupo único e irrepetible pudiéndose establecer que existe un conocimiento del tema por las vivencias y la cotidianidad, aunque algunos desconozcan el soporte teórico y los documentos existentes, hacen parte del proceso educativo que tiene como objetivo la transformación del individuo sin distingo alguno.

Se puede deducir que en la cotidianidad del quehacer docente siempre surgirán dificultades durante el proceso de formación para los estudiantes, esto es un resultado de la falta de coherencia que hay en las políticas educativas que establecen modelos de alto nivel de exigencia, olvidando las poblaciones menos favorecidas, es necesario ajustar la normatividad para que al aplicarlo se convierta en procedimientos institucionales que permitan dar solución en gran parte a problemas de alimentación, desplazamiento, participación en eventos académicos con 
ánimo de lucro, conectividad, actividades extracurriculares que por insignificantes que parezcan representan una inversión de dinero y en algunos casos son inalcanzable, todo lo anterior son elementos utilizados como variables de calificación, desconociendo los problemas económicos, familiares, personales y que en muchas ocasiones las consecuencias son marginalidad educativa y la deserción.

Se puede establecer que al llevar a cabo el proceso de enseñanza por parte de los docentes de la facultad de ciencias empresariales, por el diseño establecido de las asignaturas no se tiene en cuenta elementos tales como el lugar de la ubicación de residencia de los estudiantes, la condición socio económica, su núcleo familiar y el acceso a la información, en donde dichas variables pueden establecer una diferencia en las posibilidades de obtener un mejor desempeño académico por parte del individuo; pero, es de comprender que las orientaciones nacionales están direccionadas al perfeccionamiento de las habilidades y destrezas que pueda adquirir el sujeto para el desarrollo de una profesión, indistintamente de su procedencia, nivel social y cantidad de recursos que posea, donde todos los estudiantes tienen el mismo escenario y se presume por parte del gobierno que estas condiciones propician la igualdad, olvidándose de la equidad para ofrecerle más a quien más lo necesite.

\section{Referencias}

[1] C. Crosso Silva. "El derecho a la educación de personas con discapacidad impulsando el concepto de educación inclusiva", Revista Latinoamericana de Educación Inclusiva, vol. 4, no. 2, pp. 65-82, 2010.

[2] A. Sales Ciges. "La formación intercultural inclusiva del profesorado: Hacia la transformación social", Revista Latinoamericana de Educación Inclusiva, vol. 4, no. 1, pp. 79-95, 2010 .

[3] C. Jiménez Fernández and M. A. González Galán. Pedagogía diferencial y atención a la diversidad. Madrid: Universitaria Ramón Areces, 2011.

[4] E, Morín. La Vía. Para el futuro de la Humanidad. Barcelona: Paidós, 2011.

[5] H. Giroux. Teoría y Resistencia en Educación. Una pedagogía para la oposición. Argentina: Siglo Veintiuno Editores, 2004.

[6] P. Freire. Pedagogía de la Autonomía: Saberes necesarios para la práctica educativa. Sao Paulo: Paz e Terra, S.A, 2004.

[7] J. Viaña. La Interculturalidad como Herramienta de Eman- cipación. Hacia una redefinición de la Interculturalidad y sus Usos Estatales. Instituto Internacional de Integración Convenio Andrés Bello. La Paz-Bolivia: Campos Iris S.R.L, 2009.

[8] X. Besalú. "Corrientes y tendencias en la educación intercultural" Presentado en: Seminario Teoría y práctica de la educación intercultural en la Universidad Complutense de Madrid, 2004.

[9] M. Samaniego Sastre. Condiciones y Posibilidades de las Relaciones Interculturales: Un proceso Incierto. Fundación CIDOB, Barcelona, 2005.

[10] B. Mendoza. "Las competencias interculturales. Aproximaciones críticas y analíticas a la construcción de sus significados dentro de la Universidad Veracruzana intercultural, sede totonacapan" Tesis de magister no publicada. Universidad Veracruzana, México, 2013.

[11] P. Arnáiz Sánchez. Educación inclusiva: una escuela para todos. Málaga: Ediciones Aljibe, 2003.

[12] E. Chiner. Percepciones y actitudes del profesorado hacia la inclusión del alumnado con necesidades educativas especiales como indicadores del uso de las educativas inclusivas en el aula. Tesis Doctoral en Educación. Universidad de Alicante. Departamento de Psicología de la Salud. España. 2011.

[13] F. Sarlo. La rurbanidad ausente. En: Comunicación y rurbanidad: aportes de y para la teoría y las practicas. Argentina: Universidad Nacional de Rio cuarto, 2009.

[14] C. Rama. Los nuevos estudiantes latinoamericanos en el siglo XXI. "La Universidad se Reforma IV, los estudiantes". ORUS, IESALC, UPEL, UCV, pp. 9-25, Caracas, 2006.

[15] O. Díaz Haydar and F. Franco Media. "Percepciones y actitudes de los docentes hacia la inclusión educativa en Soledad, Atlántico” Zona Próxima no. 12, pp. 12-39, 2010.

[16] R. Hernández, C. Fernández and P. Baptista. Metodología de la investigación. México: Mac Graw-Hill, 2003.

[17] F. Alvira. El análisis de la realidad social. Métodos y técnicas de investigación, 1a. reimpresión, 2a. edición. Madrid: Alianza Universidad Textos, 1996.

[18] M. Tamayo. El proceso de investigación científica. México: Limusa, 1998.

[19] C. Méndez. Metodología diseño y desarrollo del proceso de investigación con énfasis en ciencias empresariales, 4a. edición. México: Limusa, 2009.

[20] F. Arias. El proyecto de investigación: Introducción a la metodología científica. Caracas: Episteme, 2004. 